\title{
Med.Pix \\ Itchy bumps on the back
}

Richard P Usatine

Section Editor, wjm

University of California,

Los Angeles

200 Medical Plaza, Ste

220

Los Angeles, CA

90095-1628

Correspondence to:

Dr Usatine

rusatine@ucla.edu

West J Med

2000;172:366-367
QUESTION: A 51-year-old woman is seen because of a 3-day history of itchy bumps on her back. She says that she awakened 3 days ago with itching on her back and noticed red bumps on her back only. She has no idea how this rash started and has no previous history of a similar rash. She is otherwise in good health, has no fever, chills, or other systemic symptoms, and is not taking any medications. She has no history of allergy to medications, foods, or other substances. She says she has not had any unusual foods and has not started to use any new soaps, laundry detergents, or topical preparations. She has not worn any new clothing in the past week. She has not gone camping or hiking in the past month.

Examination reveals an erythematous papular eruption with possible pustules confined to the back (see figure). There were no burrows between the fingers, no nail changes, and no scaling.

What essential question remains to be asked to make this diagnosis? What is the appropriate treatment of this rash?

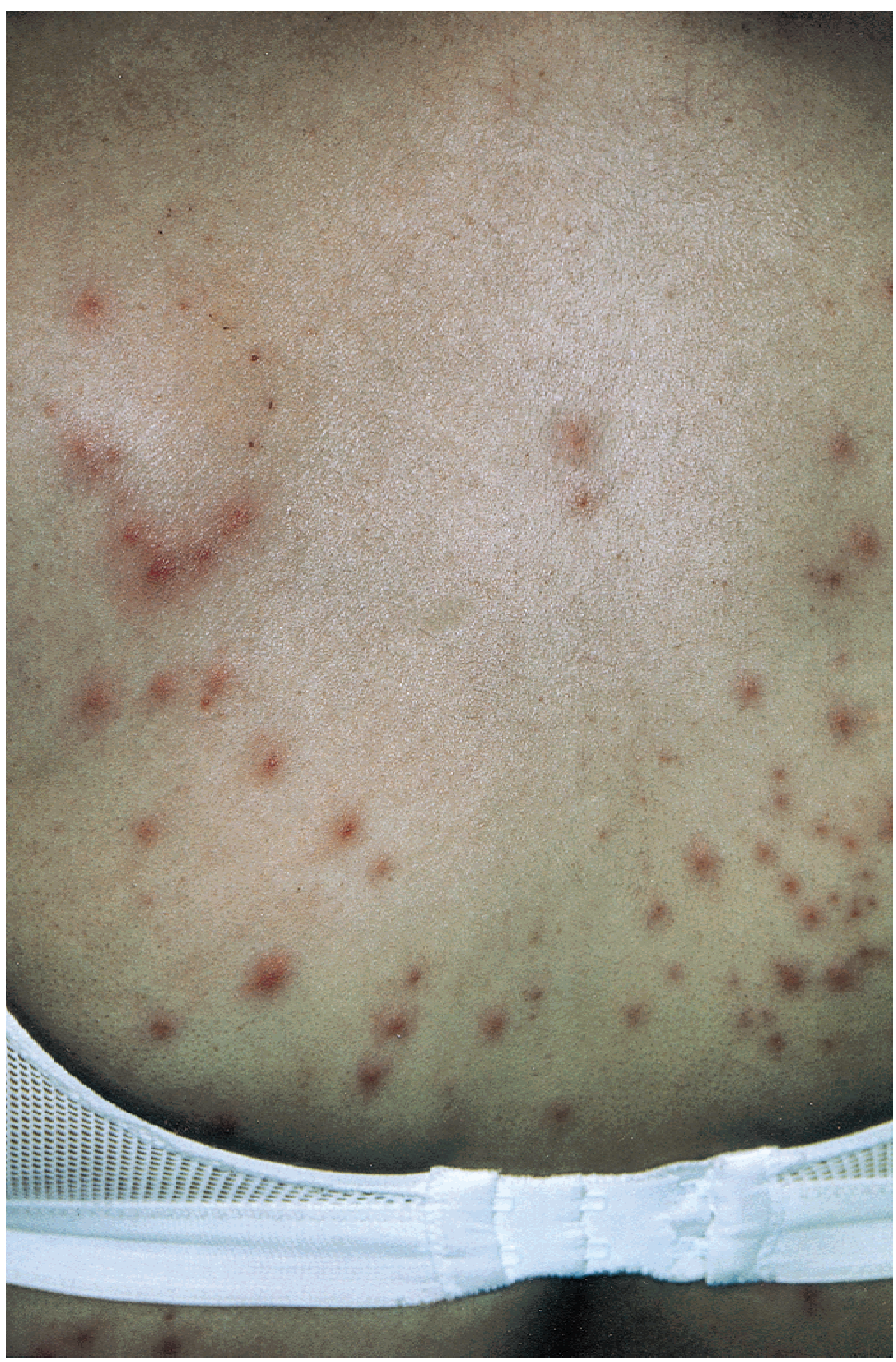

Itchy red bumps were on the back only 
ANSWER: Careful examination with an optical loupe showed that the lesions were perifollicular, and some were pustular. The essential question was asked: "Have you recently spent time in a hot tub or spa?" The patient replied in wonderment, "How did you know that, and what does a hot tub have to do with this rash?" In fact, she said that she had been in her friend's hot tub twice just before this rash broke out. She was unaware if any of her other friends had a rash.

The patient has Pseudomonas folliculitis, which is also called "hot tub folliculitis." Although this is usually caused by $P$ aeruginosa, a culture is not needed to establish this diagnosis. This can be a difficult diagnosis to make unless the pattern is recognized and the essential question asked about exposure to a hot tub. Other sources of this infection include contaminated loofah sponges and diving suits.

The differential diagnosis for a pruritic erythematous papular eruption is vast. Some of the more common possibilities include viral exanthemas; scabies; various fungal infections; and allergic reactions to medication, foods, soaps, laundry detergents, topical preparations, plants, or clothing. The use of magnification to determine that the lesions were visible around hair follicles and that some were pustular helped to make this diagnosis. The primary morphologic characteristics of Pseudomonas folliculitis include papules, pustules, urticarial plaques, macules, and vesicles.

Folliculitis can also be caused by other bacterial organisms, such as Staphylococcus aureus and Streptococcus pyo- genes. A specimen may be taken from some pustules for bacterial culture if the causative agent is uncertain. If the rash is scaly, a scraping for a potassium hydroxide preparation may be helpful to look for Pityrosporum species (a superficial yeastlike organism that can cause folliculitis).

\section{TREATMENT}

In most cases, folliculitis caused by Pseudomonas aeruginosa will resolve spontaneously within 10 days. ${ }^{1}$ In severe cases, an oral antipseudomonal antibiotic, such as a fluoroquinolone, may be prescribed. In this case, the physician and patient decided together to allow the folliculitis to resolve on its own. The patient did not want to use an antibiotic unless it was absolutely necessary. The physician suggested that the patient might use oral diphenhydramine hydrochloride (Benadryl) to treat the itching, if needed. Prevention requires meticulous cleaning of the hot tub and appropriate water chemical management.

The patient called the physician a week later to report that the rash had disappeared, but some residual discoloration still remained. The physician reassured her that the residual discoloration, which is due to postinflammatory hyperpigmentation, should fade during the coming months.

Reference

1 Habif T. Clinical Dermatology: A Color Guide to Diagnosis and Therapy. 3rd ed. St Louis, MO: Mosby; 1996.

\section{How to submit your medical images for publication in Med.Pix}

Do you have images (slides, photos, etc.) of compelling visual cases in clinical medicine?

If other primary care providers would find them interesting, we would like to publish them, along with a brief description of the clinical presentation and a question for the readers. The answer should provide an explanation of the clinical case along with information on differential diagnosis and treatment. The treatment section should suggest an evidence-based approach and be supported by current references. Multiple images may be submitted, and interesting connections between these images may be highlighted.

High-quality slides, illustrations, and photographic prints may be mailed to:

Richard Usatine, MD; Section Editor, Med.Pix; 200 UCLA Medical Plaza, Suite 220 Los Angeles, CA 90095-1628; Fax 310-206-0181.

Written descriptions and electronic images may be submitted to: rusatine@mednet.ucla,edu.

Note: If the patient's identity is recognizable, a signed permission form should accompany the submission materials.

Teen passengers are a hazard to the driver Teenage drivers are most likely to be killed when they have a car full of passengers, according to an analysis of routine data collected by the US government (JAMA 2000;283:1578-1582). Carrying three or more passengers triples a teenager's risk of a fatal accident, a risk that is already much higher than that of older drivers. The researchers speculate that peers in the back seat provoke riskier driving, including speeding, skidding, jumping red lights, and drinking. 